\title{
An antibiotic cement-coated locking plate as a temporary fixation for treatment of infected bone defects: a new method of stabilization
}

Chao Jia ${ }^{1 \dagger}$, Xiaohua Wang ${ }^{1 \dagger}$, Shengpeng $Y u^{1}$, Hongri Wu${ }^{1}$, Jie Shen ${ }^{1}$, Qiang Huang ${ }^{2}$ and Zhao Xie

\begin{abstract}
Background: The induced membrane technique has achieved good clinical results in the treatment of infected bone defects, and external fixation is the main method, but it causes inconvenience and complications in patients. In this study, our objective was to investigate the outcomes of using an antibiotic cement-coated locking plate as a temporary internal fixation in the first stage of the surgical induced membrane technique for treating extremities with infected bone defects.
\end{abstract}

Methods: We retrospectively analysed patients with lower extremity infected bone defects in our department between January 2013 and December 2017. All patients were treated with the induced membrane technique. In the first stage, the defects were stabilized with an antibiotic cement-coated locking plate as a temporary fixation after debridement, and polymethyl methacrylate cement was implanted to induce the formation of a membrane. In the second stage, bone grafting rebuilt the bone defects after infection control, and the temporary fixation was changed to a stronger fixation.

Results: A total of 183 patients were enrolled, with an average follow-up duration of 32.0 (12-66) months. There were 154 males and 29 females with an average age of 42.8 (10-68) years. The infection sites included 81 femurs, 100 tibias and 2 fibulas. After the first stage of treatment (infection control), 16 (8.7\%) patients had recurrence of infection. In terms of the incidence of complications, 4 patients had poor wound healing, 2 patients had fixation failure and 1 patient had femoral fracture due to a fall. After the second stage of treatment (bone reconstruction), there were 24 (13.1\%) recurrences of infection, with a mean time of 9.9 months (range 0.5 to 36). Among them, 18 patients underwent bone grafting after re-debridement, 6 received permanent placement of antibiotic bone cement after debridement and 2 patients refused further treatment and chose amputation. Bone healing was achieved in 175 (95.9\%) patients at the last follow-up, and the average time to bone union was 5.4 (4-12) months.

Conclusions: Antibiotic cement-coated locking plates have good clinical effects in the control of bone infection, but attention must be paid to the possible difficulty of skin coverage when applied in calves.

Keywords: Bone infection, Infected bone defects, Internal fixation, Antibiotic cement-coated locking plate

\footnotetext{
* Correspondence: xiezha054981@163.com

${ }^{\dagger}$ Chao Jia and Xiaohua Wang contributed equally to this work.

'Department of Orthopedics, First Affiliated Hospital, Army Medical University

(Third Military Medical University), Chongqing 400038, People's Republic of

China

Full list of author information is available at the end of the article
}

(c) The Author(s). 2020 Open Access This article is distributed under the terms of the Creative Commons Attribution 4.0 International License (http://creativecommons.org/licenses/by/4.0/), which permits unrestricted use, distribution, and reproduction in any medium, provided you give appropriate credit to the original author(s) and the source, provide a link to the Creative Commons license, and indicate if changes were made. The Creative Commons Public Domain Dedication waiver (http://creativecommons.org/publicdomain/zero/1.0/) applies to the data made available in this article, unless otherwise stated. 


\section{Introduction}

Bone infection is a major problem in the department of orthopaedics. Sufficient stabilization and thorough debridement are conducive to the treatment of infection. The external fixator is a widely accepted form of fixation that provides stability in the treatment of bone infections [1], which can not only provide stability but also prevent the formation of bacterial biofilms, which always increase the recurrence of infection [2]. However, external fixation has many complications, such as pin track infection [3], poor stability, joint stiffness and adverse effects on daily life. To avoid these deficiencies and shortcomings, some scholars have attempted to use internal fixations in bone infection. Liporace [4] and Conway [5] attempted to treat infected bone fractures with antibiotic cement-coated locking plates. However, the study had few cases and short follow-up times, and further analysis of the long-term efficacy and application conditions or complications is incomplete. In this study, we report a series of patients with extremity infections treated with antibiotic cement-coated locking plates as internal fixation in the first stage of the induced membrane technique, followed by grafting to rebuild bone defects after formation of the induced membrane. Our aim was to investigate the outcomes of this new method and to provide a reference for clinicians when applying this method.

\section{Patients and methods}

This study was approved by the ethics committee of our hospital, and informed consent was obtained from the patients for the publication of individual clinical details and accompanying images. We retrospectively analysed patients with lower extremity infected bone defects in our department between January 2013 and December 2017. Diagnosis of bone infection was made based on local bone pain, rubor and localized swelling, or a draining sinus tract during examination, imaging procedures, microbiological and histopathological examinations and laboratory studies [6]. Our inclusion criteria were as follows: (1) lower extremity bone infection patients admitted to our department; (2) treatment with the induced membrane two-stage technique, with antibiotic cementcoated locking plates used as temporary internal fixation after debridement; (3) bone defects remaining after debridement; and (4) follow-up time > 12 months. The exclusion criteria were as follows: (1) malignant tumours; (2) serious vascular and nerve injuries with no possibility of limb salvage; and (3) incomplete follow-up data.

\section{Surgical technique}

In the first stage, thorough debridement was performed, and a locking plate coated with antibiotic cement was used to stabilize the bone defects. The bone defects were filled with antibiotic bone cement to induce the formation of the induced membrane. In the second stage, bone grafting was performed to rebuild bone defects.

\section{Stage I (infection control)}

$\mathrm{X}$-ray, MRI and radionuclide examination were used to determine the approximate infectious area. The sinus, sequestrum, necrotic tissue and ischaemic sclerotic bone were thoroughly eradicated. Aggressive debridement utilising a high-speed saline-cooled burr removed necrotic bone, granulation tissue was eliminated with a rongeur, and the membrane curetted off the bed of bone. All infected and nonviable bone and soft tissue was cleared until good punctate haemorrhage appeared (characterized by a "paprika sign"). Specimens of infected bone and infected tissue were sent for pathological examination and microbiological examination. The wound was irrigated with a large amount of iodophor, hydrogen peroxide and saline. A locking compression plate was used to stabilize the femur, and a reconstruction locking plate was used to stabilize the tibia and fibula. Antibiotic cement ( $0.5 \mathrm{~g}$ of gentamicin per $40 \mathrm{~g}$ of PMMA) (Heraeus, Hanau, Germany) mixed with $5 \mathrm{~g}$ of vancomycin was used to fill the bone defects. The plate was wrapped and extended $1-2 \mathrm{~cm}$ to the proximal and distal ends to expand the volume of the induced membrane. Free flaps or local transfer flaps were used for some patients to ensure full wound coverage. Drainage tubes were placed before suturing. Effective intravenous antibiotics were administered after operation based on the sensitivity of the isolated bacteria, and a third-generation cephalosporin (ceftazidime) was administered if no bacteria were isolated. Patients were encouraged to exercise in bed. If infection recurred, the first-stage operation was performed repeatedly.

\section{Stage II (bone reconstruction)}

At least 6 weeks after the first stage of treatment, when the soft tissue condition was good, there were no clinical signs of infection (swelling, fever, pain, sinus formation and so on); blood tests (white blood cells (WBCs), Creactive protein (CRP), and erythrocyte sedimentation rate (ESR)) returned to normal; and the imaging examination did not indicate infection, so the second stage of treatment was feasible. The former incision was chosen, and the induced membrane was cut longitudinally to carefully protect the integrity of the induced membrane. The bone cement and internal fixation were removed thoroughly, and the bone ends were decorticated. Autografts were harvested from the iliac crest (monolateral or bilateral), and allografts were mixed for patients with large bone defects. An intramedullary nail, a locking plate or an intramedullary nail attached to a locking plate was selected for final stabilization. The induced 
membrane was sutured carefully, and then the incision was sutured completely.

\section{Postoperative follow-up}

Follow-up was performed every month after the first stage and every 3 to 6 months after the second stage. The review included clinical symptoms and signs of infection (such as swelling, fever, pain and sinus formation), laboratory examinations (WBC, ESR, CRP) and anteroposterior and lateral X-ray examination. The recurrence of infection, bone healing and complications were observed and recorded. When the WBC, ESR and CRP returned to normal and there were no clinical signs of local infection (such as swelling, fever, pain and sinus formation), no recurrence of infection was considered to have occurred. The bone union time was obtained by Xray, and radiological union was defined as three-sided cortical bridging on two perpendicular X-rays of the defect zone [7].

\section{Statistics}

Data analysis was performed using SPSS 23.0 statistical software (SPSS Inc., USA). The measurement data is represented with ranges, and the enumeration data are expressed by rate. Enumerated data were compared using Pearson or Fisher's exact tests. The difference between the groups was considered to be significant when $p<0.05$ in a two-sided test.

\section{Results}

A total of 183 patients with bone infection were enrolled. There were 154 males and 29 females with an average age of 42.8 (10-68) years. The mean follow-up time after bone reconstruction was 32.0 (12-66) months. The infection sites included 81 femurs, 100 tibias and 2 fibulas. According to the aetiological classification proposed by Waldvogel [8], there were 35 cases of haematogenous osteomyelitis, 148 cases of post-traumatic osteomyelitis of which 54 had secondary to internal fixation of closed fractures and the other 94 had secondary to open fractures. According to the Cierny-Mader anatomic classification [1], there were 48 patients with type III and 135 patients with type VI. According to the Cierny-Mader physiologic classification [1], there were 109 patients of class A and 85 patients of class B. The duration of bone infection before admission ranged from 0.5 to 540 months. The average length of bone defected was $7.7(1.5-22.7) \mathrm{cm}$. Among the 183 patients, 113 (61.7\%) had positive bacterial isolations. Among those 113 patients, 92 had cases of single bacterial infections, and 21 had cases of mixed bacterial infections. Of these, 56 (49.6\%) were infected with Staphylococcus aureus (including 16 with methicillin-resistant Staphylococcus aureus (MRSA), 13 with Enterobacter cloacae, 11 with Pseudomonas aeruginosa and 9 with Escherichia coli. However, 69 patients had negative bacterial cultures (data shown in Table 1).

Table 1 Patients' data from the 183 patients

\begin{tabular}{|c|c|c|}
\hline \multicolumn{2}{|l|}{ Number (male-to-female), $n$} & $183(154: 29)$ \\
\hline \multicolumn{2}{|l|}{ Mean age (range), years } & $42.8(10-68)$ \\
\hline \multicolumn{2}{|l|}{ Site (femur; tibia; fibula), $n$} & $81 ; 100 ; 2$ \\
\hline \multicolumn{2}{|l|}{ Duration of bone infection (range), months } & $0.5-540$ \\
\hline \multirow[t]{4}{*}{ Aetiology (no. of patients) } & Post-traumatic & 148 \\
\hline & Closed fractures & 54 \\
\hline & Open fractures & 94 \\
\hline & Haematogenous & 35 \\
\hline \multirow[t]{5}{*}{ Organisms (no. of patients) } & Staphylococcus aureus (MRSA) & $56(16)$ \\
\hline & Enterobacter cloacae & 13 \\
\hline & Pseudomonas aeruginosa & 11 \\
\hline & Escherichia coli & 9 \\
\hline & Negative & 69 \\
\hline Cierny-Mader classification & III & 48 \\
\hline (no. of patients) & IV & 135 \\
\hline \multirow[t]{2}{*}{ Physiologic class } & A & 103 \\
\hline & $\mathrm{B}$ & 80 \\
\hline Smoker & & 70 \\
\hline Follow-up (12-66 months) & $\begin{array}{l}\text { Extra revision } \\
\text { Infection-free bone healing }\end{array}$ & $\begin{array}{l}26 / 183 \\
175 / 183\end{array}$ \\
\hline
\end{tabular}


After the first stage of treatment for infection control, 16 (8.7\%) patients had recurrence of infection; 12 patients underwent two debridements, and 4 patients underwent three debridements before the infection was controlled. Complications occurred in 7 (3.8\%) patients, including delayed union of the incision in 4 patients with a tibia infection; complications related to internal fixation in 3 patients: a femoral fracture resulting from an accidental fall in 1 patient and screw loosening in 2 patients with a long segmental bone defect of the femur. There was a significant difference in the recurrence rate between type A (4/ $103)$ and type $B(12 / 68)$ patients after the first stage of treatment $(p=0.008<0.05)$. There was no significant difference in the recurrence rate among different sites of bone infection $(p=0.220>0.05)$ (data shown in Table 2).

After the second stage of treatment for bone reconstruction, there was recurrence of infection in 24 (13.1\%) patients. Bone reconstruction was not performed in 6 elderly patients, and permanent antibiotic bone cement was implanted after radical debridement. Two patients refused further treatment and opted for amputation. Eighteen patients repeatedly underwent debridement and bone grafting. Finally, 175 patients $(95.6 \%)$ achieved radiographic bone union within an average of 5.4 (4-12) months. (Typical cases are presented in Figs. 1, 2, 3 and 4).

\section{Discussion}

Bone infection is one of the most challenging conditions in the field of orthopaedics. Its essence is a bacterial biofilm [9], which can resist host immunity and antibiotics $[10,11]$. Bacteria easily attach to the surface of the metal surface to form biofilms, leading to infection and relapse $[12,13]$. Therefore, internal fixation is not recommended in the presence of bone infections. As a widely accepted instrument, an external fixation can span the infected area and avoid the exposure to metal foreign bodies [14]. However, pin tract infection is the major complication of external fixation, which can decrease the stability [3]. When the infected site is close to the joint, transarticular fixation leads to joint stiffness [15], which is

Table 2 Possible predictors of revision after first-stage treatment

\begin{tabular}{|c|c|c|c|}
\hline Group (n) & Recurrent revision (n) & Infection controlled $(n)$ & $p$ value \\
\hline Total (183) & 16 & 167 & \\
\hline \multicolumn{4}{|l|}{ Gender } \\
\hline Male (154) & 15 & 139 & \multirow[t]{2}{*}{0.458} \\
\hline Female (29) & 1 & 28 & \\
\hline \multicolumn{4}{|l|}{ Age } \\
\hline$<40$ years $(88)$ & 7 & 81 & \multirow[t]{2}{*}{0.716} \\
\hline$\geqq 40$ years $(95)$ & 9 & 86 & \\
\hline \multicolumn{4}{|l|}{ Physiologic class } \\
\hline Type A (103) & 4 & 99 & \multirow[t]{2}{*}{0.008} \\
\hline Type B(80) & 12 & 68 & \\
\hline \multicolumn{4}{|l|}{ Aetiology } \\
\hline Post-traumatic (148) & 12 & 136 & \multirow[t]{2}{*}{0.770} \\
\hline Haematogenous (35) & 4 & 31 & \\
\hline \multicolumn{4}{|l|}{ Classification } \\
\hline Type III (48) & 4 & 44 & \multirow[t]{2}{*}{1.000} \\
\hline Type IV (135) & 12 & 123 & \\
\hline \multicolumn{4}{|l|}{ Location } \\
\hline Femur (81) & 7 & 74 & \multirow[t]{3}{*}{0.220} \\
\hline Tibia (100) & 8 & 92 & \\
\hline Fibula (2) & 1 & 1 & \\
\hline \multicolumn{4}{|c|}{ Number of previous operations } \\
\hline$\geq 1(165)$ & 13 & 152 & \multirow[t]{2}{*}{0.519} \\
\hline$<1(18)$ & 3 & 16 & \\
\hline \multicolumn{4}{|l|}{ Smoker } \\
\hline Yes (70) & 9 & 61 & \multirow[t]{2}{*}{0.121} \\
\hline No (113) & 7 & 106 & \\
\hline
\end{tabular}






Fig. 1 Twenty-one-year-old male suffered from bone infection of the left femur for 2 years. The result of bacterial culture during operation was Enterobacter cloacae. a X-ray shows bone destruction. b Resection of the lesion; antibiotic cement filled the defect and wrapped the plate. c Bone graft after 8 weeks. $\mathbf{d}$ Callus growth was observed after 3 months. e Bone union after 24 months

not conducive to the recovery of joint function. External fixation is bulky, which affects patients' ordinary life [16]. Long-term placement of the external fixation also has a negative impact on patients' mental health [17]. Mark [18] reported 11 cases of femur infection treated with plate internal fixation after complete debridement. Alemdar [19] reported that 15 cases of deep infection after lower extremity fracture were treated with "non-contact" plate internal fixation on the basis of complete debridement, but no matter how thorough the debridement was, bacteria still existed [20], and residual bacteria could form biofilms on the surface of the internal fixation, resulting in recurrence of infection.
Wrapping with antibiotic bone cement can prevent bacteria from adhering to the surface of the internal fixation, and the high concentrations of antibiotics released from antibiotic bone cement can kill the residual bacteria after debridement, preventing the internal fixation from becoming a new source of infection [21, 22]. Our research applied internal fixations with locking plates coated with antibiotic bone cement for the treatment of 183 patients with lower extremity infection. The infection control rate was 91.3\% after the first debridement. It has been proven from clinical practice that internal fixation can be used after infection debridement, which breaks through the prohibition of internal fixation in the

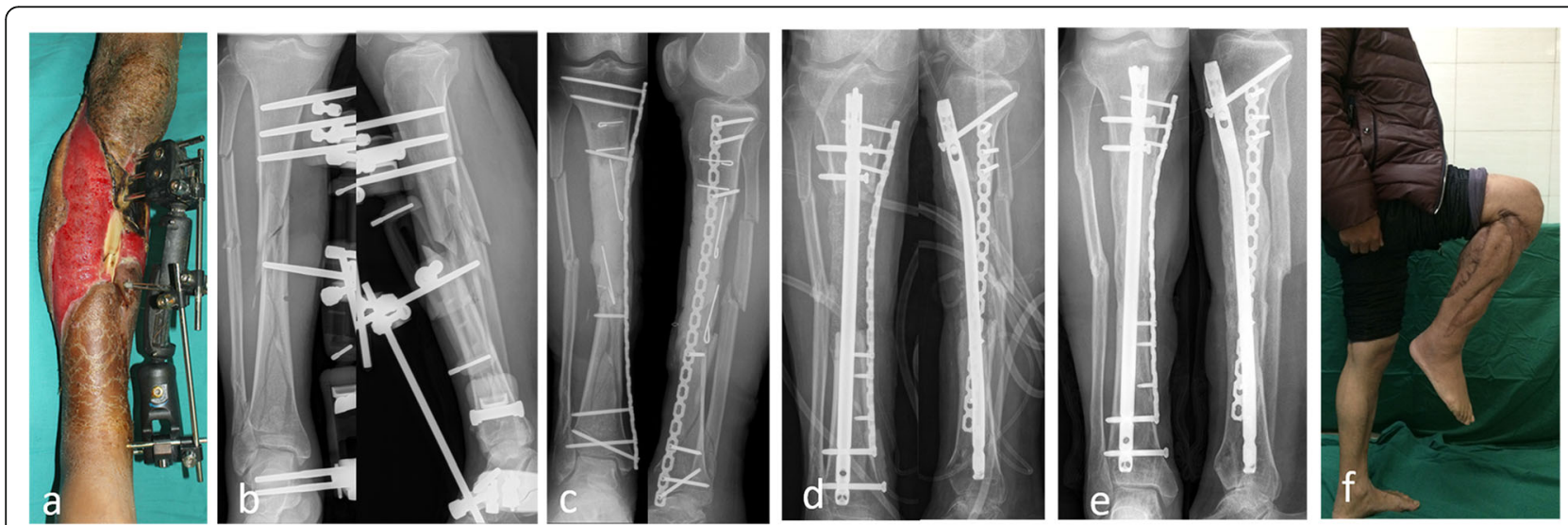

Fig. 2 Forty-seven-year-old male. Bone infection after open injury. The culture results showed Pseudomonas aeruginosa. a Extensive soft tissue defect and bone exposure. $\mathbf{b}$ Preoperative X-ray. c Resection of the lesion. Antibiotic cement filled the defect and wrapped the plate. $\mathbf{d}$ Bone graft after 4 months. e Bone union after 24 months. f Function well 

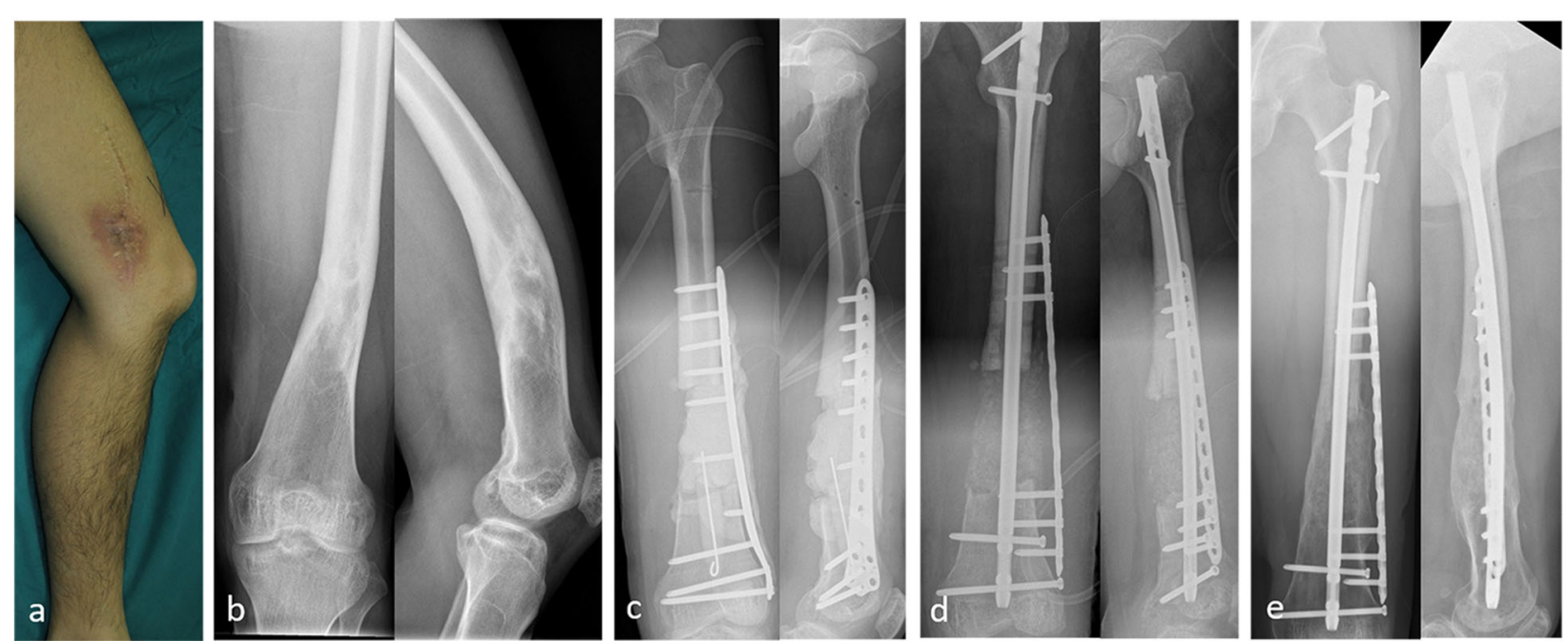

Fig. 3 Thirty-year-old male. Bone infection of the right femur for 18 years. The culture results showed Staphylococcus aureus. a Preoperative photo. $\mathbf{b}$ Preoperative $\mathrm{X}$-rays. $\mathbf{c}$ X-rays after the first stage. $\mathbf{d}$ X-rays after the second stage. e Bone union after 24 months

case of bone infection. This method did not seem to cause more infectious complications compared to external fixation, but more importantly, patients were comfortable and complications associated with external fixation were avoided. Thorough removal of infected bone and soft tissue is the prerequisite for the use of internal fixation, and it is also the core for bone infection treatment [23, 24]. The goal of debridement is to achieve a viable vascularized environment and restore local immunity. The local high concentrations of antibiotics also play a key role. Antibiotics added to bone cement are slowly released over time. A high dose of antibiotics surrounds the plate to achieve a minimum local inhibitory concentration. These measures can prevent biofilm formation and thus reduce the infection recurrence rate [22, 25-27]. Furthermore, good stability is beneficial to the treatment of infection $[28,29]$. It was confirmed in animal experiments that the infection rate in the unstable group was more than twice as high as that in the stable group [30]. The locking plate provides angular stability and axial stability [31], which provides excellent anchoring in osteoporotic bone. It can also help protect joint function by avoiding transarticular fixation when bone infection occurs in the metaphysis. In addition, the locking plate does not contact the bone cortex and helps to protect the periosteal blood supply.

In this study, all patients were treated with antibiotic cement-coated locking plates. After the first stage of treatment, $16(8.7 \%)$ patients experienced recurrence of infection, which manifested as wound nonunion, sinus formation, local redness, swelling and pain. After repeated debridement and internal fixation, the infection was controlled, and bone grafting progressed smoothly. We found that the recurrence rate of type B (17.6\%) patients after debridement was significantly higher than that of type A patients $(3.9 \%)(p<0.05)$. This finding is consistent with Simpson's prospective study. Simpson [32] found that patients defined as type $B$ had a higher recurrence rate than patients defined as type $\mathrm{A}$ and that type $\mathrm{B}$ patients were recommended for more extensive debridement.

Delayed local skin healing occurred in the tibia of 4 patients. Due to long-term infection and repeated operations, the local soft tissue condition was poor, and the soft tissue defect after debridement made the incision suture tension larger. Therefore, skin flaps are recommended to ensure tension-free suturing of the incision. Reconstruction of the soft tissue envelope and establishment of a good blood supply can enhance local anti-infection capacity. Systemic antibiotics are more likely to be effective locally. Limb elevation and anti-swelling drugs were used after the operation. Fracture or loosening of the internal fixation is related mainly to weight bearing and accidental falls. In the first stage of treatment, the goal of the locking plate is to provide enough stability to allow patients to have a comfortable outpatient experience while controlling infection and to perform functional exercises. Considering that long plates will increase the difficulty of infection treatment, we chose a shorter plate, which may not be strong enough. Therefore, patients are recommended to walk with crutches without weight-bearing or partial weightbearing after the first stage. 

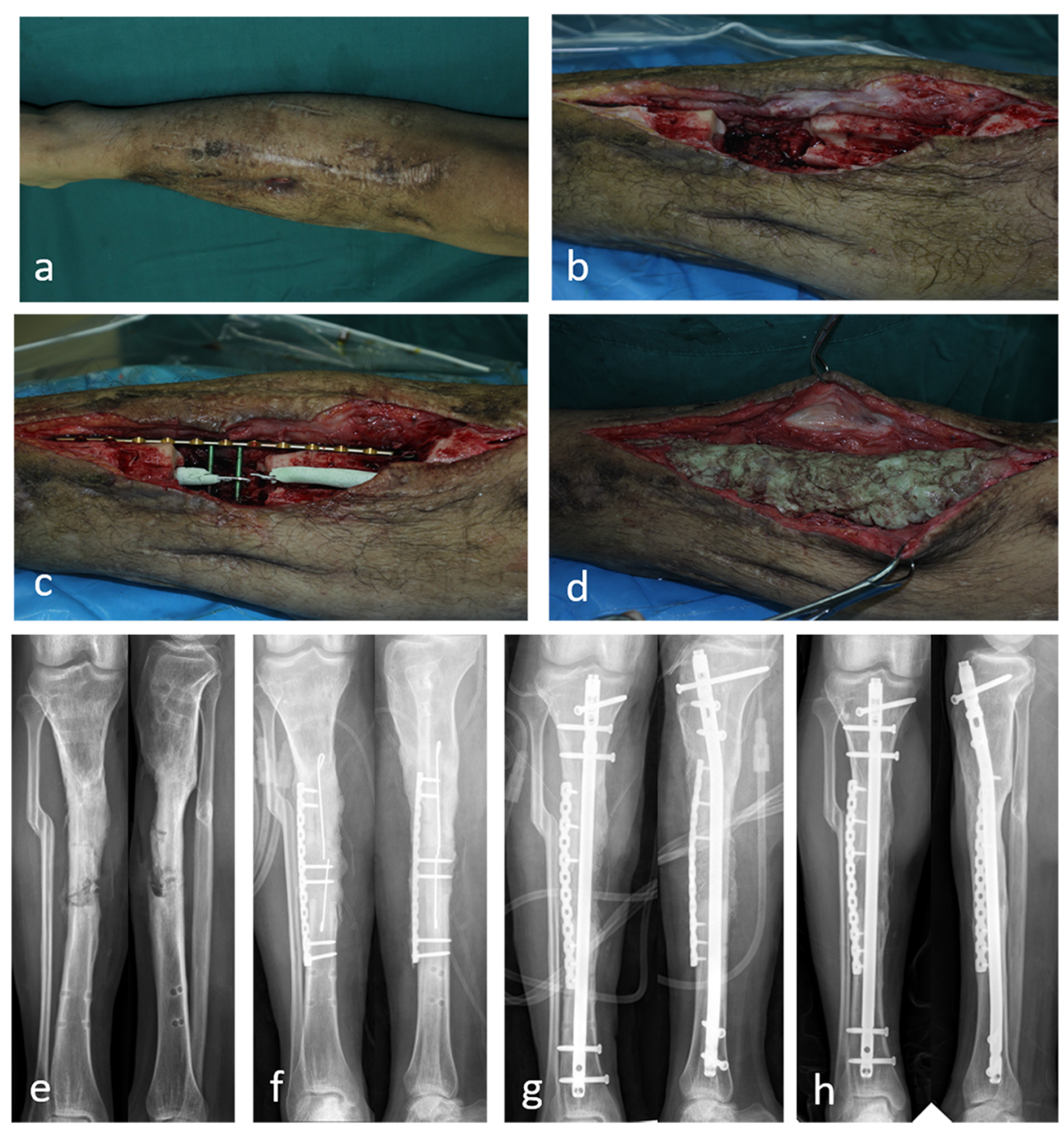

Fig. 4 Twenty-three-year-old male. Bone infection after open reduction and internal fixation for 3 years. Deep tissue culture revealed Staphylococcus aureus. a Preoperative photo. $\mathbf{b}$ After debridement. c Reconstructive locked plate fixation. $\mathbf{d}$ Antibiotic bone cement filled with the defects and wrapped the fixation. e Preoperative X-rays. $\mathbf{f} X$-rays after the first stage. $\mathbf{g}$ X-rays after the second stage. $\mathbf{h}$ Bone union after 36 months

The induced membrane technique [33] proposed by Masquelet in 2000 is a new method for the treatment of bone defects. The membrane prevents the absorption of the graft bone and produces factors that promote osteogenesis [34], and the bone union rate is $80 \sim 100 \%$ [35]. In this study, 175 (95.6\%) patients obtained radiological bone union, and the union time was 5.4 months (range 4 to 12 months). When using locking plates for internal fixation, the plate should be completely wrapped in bone cement to ensure the integrity of the induced membrane and avoid possible residual bacterial adhesion. Furthermore, the coverage is extended to the bone tip to increase the volume of the induced membrane. Antibioticimpregnated cement can not only release a high concentration of antibiotics but also induce a highly vascularized membrane rich in various bone factors [36], which provides a good environment for bone reconstruction.

Although this study has a good success rate and breaks through many traditional theoretical and technical taboos, there are still some deficiencies. First, this study is a retrospective study, and it can only be summarized from clinical experience for some postoperative complications but not confirmed from the experiment. Second, there is no control group and no comparison with other established methods.

\section{Conclusion}

Temporary internal fixation with an antibiotic cementcoated locking plate has good clinical effect in the control of long bone infection in the lower extremities and is practical in all parts of the lower extremities, but the application premise must be thoroughly debrided. At the same time, poor soft tissue condition is a relative concern. Attention should be paid to the skin coverage of the lower leg.

\section{Abbreviations}

CRP: C-reactive protein; ESR: Erythrocyte sedimentation rate; MRI: Magnetic resonance imaging; MRSA: Methicillin-resistant Staphylococcus aureus; PMMA: Polymethyl methacrylate 


\section{Acknowledgments}

Not applicable.

\section{Authors' contributions}

$\mathrm{CJ}$ and XHW wrote the manuscript and performed the data collection. SPY and HRW performed the data collection and made the figures. JS and QH performed the data interpretation and helped to write the manuscript. All authors read and approved the final manuscript. CJ and XHW contributed equally to this work. ZX designed and performed the study.

\section{Funding}

This work was supported by The National Natural Science Foundation of China (81672160) and The National Key Research and Development Program of China (2016YFC1102005).

\section{Availability of data and materials}

The datasets used and analysed during the current study are available from the corresponding author on reasonable request.

\section{Ethics approval and consent to participate}

The study was authorized by the Medical Ethics Committee of Southwest Hospital (Ref. No. KY201878). All patients or patient's legal guardian gave written informed consent for inclusion in the study.

\section{Consent for publication}

Consent to publish was obtained for the patients or patient's legal guardian whose information appears in this publication.

\section{Competing interests}

The authors declare that they have no competing interests.

\section{Author details}

'Department of Orthopedics, First Affiliated Hospital, Army Medical University (Third Military Medical University), Chongqing 400038, People's Republic of China. ${ }^{2}$ Department of Orthopaedics, Traditional Chinese Medicine Hospital, Shaping Ba District, Chongqing 400038, People's Republic of China.

\section{Received: 2 December 2019 Accepted: 28 January 2020}

Published online: 11 February 2020

\section{References}

1. Cierny G 3rd, Mader JT, Penninck JJ. A clinical staging system for adult osteomyelitis. Clin Orthop Relat Res. 2003:414:7-24.

2. Giannoudis PV. Treatment of bone defects: bone transport or the induced membrane technique? Injury. 2016:47(2):291-2.

3. Parameswaran AD, Roberts CS, Seligson D, Voor M. Pin tract infection with contemporary external fixation: how much of a problem? J Orthop Trauma. 2003:17(7):503-7.

4. Liporace FA, Yoon RS, Frank MA. Use of an "antibiotic plate" for infected periprosthetic fracture in total hip arthroplasty. J Orthop Trauma. 2012;26(3):18.

5. Conway JD, Hlad LM, Bark SE. Antibiotic cement-coated plates for management of infected fractures. Am J Orthop (Belle Mead NJ). 2015;44(2):E49-53.

6. Mouzopoulos G, Kanakaris NK, Kontakis G, Obakponovwe O, Townsend R, Giannoudis PV. Management of bone infections in adults: the surgeon's and microbiologist's perspectives. Injury. 2011;42(Suppl. 5):S18-23.

7. Blum AL, BongioVanni JC, Morgan SJ, Flierl MA, dos Reis FB. Complications associated with distraction osteogenesis for infected nonunion of the femoral shaft in the presence of a bone defect: a retrospective series. The Journal of bone and joint surgery British volume. 2010;92(4):565-70.

8. Waldvogel FA, Medoff G, Swartz MN. Osteomyelitis: a review of clinical features, therapeutic considerations and unusual aspects. N Engl J Med. 1970;282(4):198-206

9. Zimmerli W, Sendi P. Orthopaedic biofilm infections. APMIS: acta pathologica, microbiologica, et immunologica Scandinavica. 2017;125(4):353-64.

10. Trampuz A, Zimmerli W. Diagnosis and treatment of infections associated with fracture-fixation devices. Injury. 2006;37(Suppl 2):S59-66.

11. Costerton JW. Bacterial biofilms: a common cause of persistent infections. Science. 1999;284(5418):1318-22.

12. Parsons B, Strauss E. Surgical management of chronic osteomyelitis. Am J Surg. 2004;188(1):57-66.
13. Evans RP, Nelson $\mathrm{CL}$, Harrison $\mathrm{BH}$. The effect of wound environment on the incidence of acute osteomyelitis. Clin Orthop Relat Res. 1993:286:289-97.

14. Mauffrey C, Hake ME, Chadayammuri V, Masquelet AC. Reconstruction of long bone infections using the induced membrane technique: tips and tricks. J Orthop Trauma. 2016;30(6):e188-93.

15. Masquelet AC, Kishi T, Benko PE. Very long-term results of post-traumatic bone defect reconstruction by the induced membrane technique. Orthop Traumatol Surg Res. 2019;105(1):159-66.

16. Wang $Y$, Jiang $H$, Deng Z, Jin J, Meng J, Wang J, Zhao J, Sun G, Qian H. Comparison of monolateral external fixation and internal fixation for skeletal stabilisation in the management of small tibial bone defects following successful treatment of chronic osteomyelitis. BioMed research international. 2017;2017:6250635.

17. Abulaiti A, Yilihamu Y, Yasheng T, Alike Y, Yusufu A. The psychological impact of external fixation using the llizarov or Orthofix LRS method to treat tibial osteomyelitis with a bone defect. Injury. 2017:48(12):2842-6.

18. Prasarn ML, Ahn J, Achor T, Matuszewski P, Lorich DG, Helfet DL. Management of infected femoral nonunions with a single-staged protocol utilizing internal fixation. Injury. 2009;40(11):1220-5.

19. Alemdar C, Azboy I, Atic R, Ozkul E, Gem M, Kapukaya A. Management of infectious fractures with "Non-Contact Plate" (NCP) method. Acta Orthop Belg. 2015;81(3):523-9.

20. Kinik H, Karaduman M. Cierny-Mader type III chronic osteomyelitis: the results of patients treated with debridement, irrigation, vancomycin beads and systemic antibiotics. Int Orthop. 2008;32(4):551-8.

21. Campoccia D, Montanaro L, Arciola CR. The significance of infection related to orthopedic devices and issues of antibiotic resistance. Biomaterials. 2006; 27(11):2331-9.

22. Hogan A, Heppert VG, Suda AJ. Osteomyelitis. Archives of orthopaedic and trauma surgery. 2013;133(9):1183-96.

23. Waldvogel FA, Papageorgiou PS. Osteomyelitis: the past decade. N Engl J Med. 1980;303(7):360-70

24. Pan C, Zhou Z, Yu X. Coatings as the useful drug delivery system for the prevention of implant-related infections. J Orthop Surg Res. 2018:13(1):220.

25. Hake ME, Young H, Hak DJ, Stahel PF, Hammerberg EM, Mauffrey C. Local antibiotic therapy strategies in orthopaedic trauma: practical tips and tricks and review of the literature. Injury. 2015;46(8):1447-56.

26. Zalavras CG, Patzakis MJ, Holtom P. Local antibiotic therapy in the treatment of open fractures and osteomyelitis. Clin Orthop Relat Res. 2004;427:86-93.

27. Thonse R, Conway J. Antibiotic cement-coated interlocking nail for the treatment of infected nonunions and segmental bone defects. Journal of Orthopaedic Trauma. 2007:21(4):258-68.

28. Patzakis MJ, Wilkins J, Wiss DA. Infection following intramedullary nailing of long bones. Diagnosis and management. Clin Orthop Relat Res. 1986;212:182-91.

29. Chen CE, Ko JY, Wang JW, Wang CJ. Infection after intramedullary nailing of the femur. Journal of Trauma-Injury Infection and Critical Care. 2003;55(2):338-44.

30. Worlock P, Slack R, Harvey L, Mawhinney R. The prevention of infection in open fractures: an experimental study of the effect of fracture stability. Injury. 1994:25(1):31-8.

31. Wagner M. General principles for the clinical use of the LCP. Injury. 2003;34:31-42.

32. Simpson AH, Deakin M, Latham JM. Chronic osteomyelitis. The effect of the extent of surgical resection on infection-free survival. J Bone Joint Surg Br. 2001;83(3):403-7.

33. Masquelet AC, Fitoussi F, Begue T, Muller GP. Reconstruction of the long bones by the induced membrane and spongy autograft. Ann Chir Plast Esthet. 2000:45(3):346-53.

34. Chotel F, Nguiabanda L, Braillon P, Kohler R, Berard J, Abelin-Genevois K. Induced membrane technique for reconstruction after bone tumor resection in children: a preliminary study. Orthop Traumatol Surg Res. 2012;98(3):301-8.

35. Morris R, Hossain M, Evans A, Pallister I. Induced membrane technique for treating tibial defects gives mixed results. Bone Joint J. 2017;99-b(5):680-5.

36. Aho OM, Lehenkari $P$, Ristiniemi J, Lehtonen $S$, Risteli J, Leskela HV. The mechanism of action of induced membranes in bone repair. J Bone Joint Surg Am. 2013;95(7):597-604.

\section{Publisher's Note}

Springer Nature remains neutral with regard to jurisdictional claims in published maps and institutional affiliations. 